\section{FRI0499 ACROMEGALY ARTROPATHY: IS THERE SOMETHING MORE BEHIND THE PAIN? A CROSS-SECTIONAL STUDY TO EVALUATE RHEUMATIC DISEASE IN GROWTH HORMONE SECRETING TUMOR PATIENTS}

M. Scarati ${ }^{1}$, S. Parisi ${ }^{2}$, N. Prencipe ${ }^{2}$, M. C. Ditto ${ }^{2}$, E. Ghigo ${ }^{2}$, E. Fusaro², S. Grottoli ${ }^{2} .{ }^{1}$ Mauriziano Hospital, Medical Department, Turin, Italy; ${ }^{2} \mathrm{AOU}$ Città della Salute e della Scienza, Turin, Italy

Background: Acromegaly is a rare disease with a remarkable impact on patients, both in terms of life expectancy and quality of life. Osteo-articular complications are one of the most frequently reported bothers. The "acromegaly artropathy" characterizes more than $70 \%$ of patients at diagnosis. Artropathy affects both spine and peripheral joints. A recent prospective study documented progression of acromegalic arthropathy identified as a worsening of osteophytes and joint space narrowing in $72-74 \%$ of patients despite long-term biochemical control. In addiction the Literature has occasionally reported cases of simultaneous presence of rheumatic diseases (rheumatoid arthritis, polymyalgia rheumatica, undifferentiated connective tissue diseases) and acromegaly and in all these cases the treatment has been delayed, because of wrong symptoms attribution to acromegaly artropathy.

Objectives: The primary goal of the study is to better characterize joint pain in acromegaly patients and to evaluate the prevalence of rheumatic disease in growth hormone $(\mathrm{GH})$ secreting pituitary tumor patients.

Methods: We enrolled 20 acromegaly subjects (AS) and 20 control subjects (CS). In each subject immunological pattern (rheumatoid factor - RF; antinuclear antibodies - ANA, ENA; anti-citrullinated protein antibodies - ACPA; erythrocyte sedimentation rate - ESR) has been evaluated; they, also, underwent bilateral joint ultrasound of hands and wrists and nail capillaroscopy. The Chi square test and the Fisher's exact test were used to evaluate the association between binary variables, while the Spearman's test to evaluate the correlation of continuous ones. A multiple or logistic regression model was calculated in order to define the association between the capillaroscopic alterations and other detected variables.

Results: Articular pain emerged as significantly more frequent in AS ( $p=$ 0.0269 ). No statistically significant differences are detected regarding immunological pattern. ANA and ENA screening resulted positive in 10\% in AS and in $5 \%$ in CS. No IgA ACPA were detected in AS or CS, while IgG ACPA were positive only in one AS subject. No significant differences were detected between $\operatorname{IgM}$ and IgG RFin the two groups (AS $5 \%$ and CS $0 \%$ ). Three fold higher IgG FR in AS compared to CS were detected. ESR levels were significantly higher than CS ( $p=0.0405)$, as well as increased power doppler (PWD) articular uptake (AS $30 \%$ vs CS $5 \%$ p 0.081). The capillaroscopic evaluation showed a significant difference in almost each parameter that has been evaluated (logistic regression: number of enlargement $p 0.004$, hemorragies $p 0.01$ and capillaries $\mathrm{p}$ 0.001), showing a moderate-severe microangiopathy in AS. Interestingly, analyzing only the acromegaly cohort, we noticed higher $\mathrm{GH}$ levels at the enrollment in patients which developed capillary enlargements $(\mathrm{GH}: 0.95 \mathrm{ng} / \mathrm{ml} \mathrm{IQ} 0,6-1,6)$

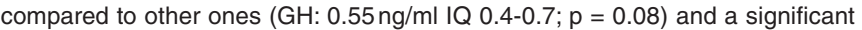
lower number of hemorrhages $(p=0.02)$ in patients treated with $G H$ antagonist pegvisomant.

Conclusion: Our results demonstrated that joint damage in acromegaly does not seem to have an autoimmune etiology. Therefore, articular damage is mechanical and increased ESR and PWD alterations seems to confirm the presence of an inflammatory component. In addition, acromegaly is characterized by a microvascular pattern of moderate-severe microangiopathy, without correlation to IGF-I, but GH levels. Although requiring further confirmatory studies, our preliminary results seem to indicate how the capillaroscopic examination could be useful to detect earlier microangiopathy and to identify patients with a greater risk of macroangiopathy development.

References:

[1] Claessen KMJA et al. Bone and joint disorders in acromegaly. Neuroendocrinology. 2016;103(1):86-95.

[2] Örük $\mathrm{G}$ et al. Is every joint symptom related to acromegaly? Endocrine. 2013 Apr;43(2):404-11.

Disclosure of Interests: None declared

DOI: 10.1136/annrheumdis-2020-eular.3134

\section{FRI0500 \\ BEHÇET SYNDROME AND CENTRAL NERVOUS SYSTEM INVOLVEMENT: A DESCRIPTIVE STUDY IN A COHORT OF PATIENTS IN OUR RHEUMATOLOGY DEPARTMENT}

N. Plaza Aulestia ${ }^{1}$, M. J. Pérez ${ }^{1}$, S. Rodríguez Montero ${ }^{1}$, J. L. Marenco ${ }^{1}$. ${ }^{1}$ Hospital Universitario de Valme, Rheumatology, Sevilla, Spain

Background: Behçet's disease (BD) is a variable vessel vasculitis characterized by mucocutaneous lesions (oral and genital ulcers). In addition, it can affect other systems such as vascular, ophthalmological, gastrointestinal tract or central nervous system (CNS). The latter being one of the most serious manifestations of Behçet's disease. We present a descriptive observational study of a cohort of patients diagnosed with Behçet's disease with CNS involvement.

Objectives: Our objective is to describe the prevalence and characteristics of various clinical manifestations in patients with Behçet.

Methods: Observational, descriptive, cross-sectional, retrospective design performed in patients with Behçet's disease in follow-up by the Rheumatology department of the Hospital de Valme, until October 2019. The following information is collected from medical records; sex, mean age at diagnosis, clinical characteristics, HLA B51 positivity and treatment.

Results: Thirty-three patients were diagnosed with Behçet's disease, with a mean age at diagnosis of $36.22+/-10.94$ years, female predominance 19 (57.57\%). Regarding the determination of HLA B 51; 5 (15.15\%) positive, 6 (18.18\%) negative and $22(66.66 \%)$ not determined. The following information about clinical manifestations is collected:

Clinical manifestations $\mathrm{n}(\%)$

Oral aphtosis

$33(100)$

Genital ulceration

Skin (Pseudofolliculitis/Erythema Nodosum) $23(69.69)$

Ocular

Anterior Uveitis

Posterior uveitis

Panuveitis

Vitritis

Retinal vasculitis

Vascular

Arthralgias/arthritis

Gastrointestina

Central Nervous System

Demyelinating lesions

Stroke

Epilepsy $12(36.36)$ $22(66.66)$ $8 / 22(36.36)$ $3 / 22$ (13.63) 3/22 (13.63) 1/22 (4.54) $1 / 22(4.54)$
$6 / 22(27.27)$ $6 / 22(27.27)$
7 (21.21) $20(60.60)$

$2(6.06)$

(6.06)

9 (27.27)

5/9 (55.55)

2/9 (22.22)

1/9 (11.11)

1/9 (11.11)

We identified 9 patients with CNS involvement; 5 demyelinating lesions (55\%), 2 strokes (22\%), 1 epilepsy/seizure (11\%) and 1 case of CNS vasculitis $(11 \%)$. Fifty-five percent of the patients were female $(55 \%)$. Two patients were positive for HLA B51 (22\%) and the rest had not been determined. Regarding treatment, all patients received both, oral and intravenous, corticosteroids (prednisone, methylprednisolone) and seven of them received DMARDs; 2 methotrexate $(28 \%), 2$ cyclosporine $(28 \%), 1$ sulfasalazine (14\%), 1 azathioprine (14\%) and 1 mercaptopurine (14\%, due to intestinal involvement). Two of them needed biologic DMARD with TNF inhibitor agents (Infliximab and Adalimumab).

Conclusion: Although neurological involvement is not common in BD, we describe higher prevalence $(22 \%)$ than other series in the literature ${ }_{1,2}$. Given the heterogeneity of the clinical presentation there is a wide differential clinical diagnosis to rule out; demyelinating diseases (multiple sclerosis), such as demyelinating lesions presented in 5 of our patients $(55 \%)$, infectious diseases (tuberculosis), other autoimmune diseases (Vogt-Koyanagi-Harada Syndrome, Eales Sd) or ischemic stroke due to atherosclerosis.

References:

[1] 10.1016/B978-0-7020-4088-7.00110-3

[2] $10.1046 / \mathrm{j} .1365-4362.2003 .01741$

[3] 10.1007/s00415-013-7209-3

Disclosure of Interests: NAHIA PLAZA AULESTIA: None declared, MARÍA JOSÉ PÉREZ: None declared, Sergio Rodríguez Montero: None declared, Jose Luis Marenco Speakers bureau: ABbvie, Pfzer, lilly DOI: 10.1136/annrheumdis-2020-eular.3281 